Orthopäde $2019 \cdot 48: 257$

https://doi.org/10.1007/s00132-019-03698-6

Online publiziert: 13. Februar 2019

(c) Springer Medizin Verlag $\mathrm{GmbH}$, ein Teil von Springer Nature 2019

CrossMark

M. Haversath ${ }^{1} \cdot$ S. Klebingat ${ }^{2} \cdot$ die VITAS-Gruppe $\cdot$ M. Jäger ${ }^{1}$

${ }^{1}$ Klinik für Orthopädie und Unfallchirurgie, Universitätsmedizin Essen, Essen, Deutschland ${ }^{2}$ Institut für Medizintechnik und Forschungscampus STIMULATE, Otto-von-Guericke-Universität Magdeburg, Magdeburg, Deutschland

\title{
Erratum zu: Abriebanalyse mit virtuellen CAD-basierten Röntgenaufnahmen in der Endoprothetik
}

\section{Erratum zu:}

Orthopäde 2018

https://doi.org/10.1007/s00132-018-

3602-Z

Sehr geehrter Leser,

sehr geehrte Leserin,

bei dem Beitrag „Abriebanalyse mit virtuellen CAD-basierten Röntgenaufnahmen in der Endoprothetik" wurde bei Herrn Klebingat leider eine fehlerhafte Affiliation angegeben.

Außerdem bitten wir folgenden Hinweis zur Förderung zu berücksichtigen:

Die Arbeit wird durch den Europäischen Fonds für Regionale Entwicklung im Rahmen der Initiative „Forschung und Entwicklung“ unter der Vorgangsnummer „ZS/2017/06/86490“ im Rahmen des Forschungscampus STIMULA$T E$ gefördert.

Wir bitten für den Fehler um Verzeihung.

Die Redaktion

\section{Korrespondenzadresse}

Prof. Dr. M. Jäger

Klinik für Orthopädie und Unfallchirurgie, Universitätsmedizin Essen

Hufelandstraße 55, 45147 Essen, Deutschland marcus.jaeger@uk-essen.de
Die Online-Version des Originalartikels ist unter https://doi.org/10.1007/s00132-018-3602-z zu finden. 